\title{
UEBER DIE WACHSARTIGE SUBSTANZ DES KOKON-SEIDENFADENS.
}

\author{
von \\ OkU MaSaml. \\ (Aus dem chemischen Laboratorium, Gunze Gesellschajt zur Herstellung \\ von Rohseiden, Ayabe, Kyōto-hì, Japan.) \\ (Eingegangen am Feb. 21., 1929.)
}

Die wachsartige Substanz von Kokonseidenfäden, deren eingehende Untersuchungen bis heute noch nicht gemacht worden sind, hat nicht nur eine physiologische Bedeutung für die Seidenwürmer, sondern übt auch einen praktischen Einfluss auf Rohseiden-industrien aus. Nach-folgende Untersuchungen beschäftigen sich hauptsächlich mit den chemischen Beschaffenheiten dieses Kokonwachses.

\section{Kurze Zusammenfassung von Versuchsresultaten.}

Kokonschichten von Bombyx mori wurden mit Aether extrahiert und eine rohe wachsartige Substanz erhalten. Die chemischen Eigenschaften derselben konnten wie unten ausgeführt zusammengefasst werden.

1.) Das Rohwachs.

Es ist ziemlich orange gefärbt wegen der Beimengung von Kokonpigment. Die getrocknete Substanz ist leicht purverisierbar. Sie bestand aus ca. $80 \%$ in siedenden Alkohol löslichen Teilen und 20\% in Alkohol unlöslichen Teilen. Es schmilzt bei $65^{\circ}$ (unkorr.). Die Elementaranalyse zeigte, C\% 77.9, $\mathrm{H} \%$ 13.2, $\mathrm{O} \% 8.9$.

Die qualitative Versuchen zeigten keinen Glyzerin und Stickstoff.

2.) Das reine Wachs.

Das Rohwachs wurde durch einmaliges Umkristallisieren aus siedendem Alkohol gereinigt und frei von Pigment gemacht. Alle unten erwähnten Versuche wurden mit diesen reinen Wachsen ausgestellt. Es bestand aus 63.14\% unverseifbarer Substanz und $36.86 \%$ von Fettsäuren. Es zeigte,

Schmelzpunkt $68^{\circ} \sim 69^{\circ}$ (unkorr.), Säurezahl 13.42, Verseifungszahl 110.77, Iodzahl 4.49, Elementarzusammensetzung $\mathrm{C} \%$ 79.4, $\mathrm{H} \%$ 13.9, $\mathrm{O} \% 6.7$.

3.) Die unverseifbaren Substanzen.

Reines Wachs bestand aus 81.92 Teilen höheren Alkohols und 18.08 Teilen Kohlenwasserstoffen. Kein Vorhandensein von Sterine wurde bestätigt, 
durch gelegentliche Farbenreaktionen von Sterine. Es schmilzt bei $84^{\circ}$ (unkorr.). Die Elementaranalyse ergab, C\% 81.6, H\% 14.6, O\% 3.8. Beim Erwärmen mit Essigsäureanhydrid ergibt sich zum grössten Teile eine Lösung und scheidet sich beim Erkalten : aber eingeringer Teil löst sich nicht und scheidet als ölige Tropfen aus siedendem Essigsäureanhydrid aus. Erstere Eigenschaften zeigten das Vorhandensein von höheren Alkoholen und die letztere das von Kohlenwasserstoffen.

4.) Die Alkohole des unverseifbaren Bestandteils.

Die Wachsalkohole und Kohlenwasserstoffe wurden voneinander durch Kochen mit Essigsäureanhydrid getrennt. Die acetilierten Alkohole zeigten,

Schmelzpunkt $67^{\circ}$ (unkorr.), Verseifungszahl 127.7, Elementarzusammensetzung $\mathrm{C} \%$ 79.35, $\mathrm{H} \% 13.25$.

\begin{tabular}{|c|c|c|c|c|}
\hline Vgl. & $\mathrm{C} \%$ & $\mathrm{H} \%$ & F.P. & Verseif.-zahl \\
\hline Cerylacetat & 79.16 & 13.30 & $65^{\circ}$ & 132.3 \\
\hline Myricylacetat & 79.92 & 13.40 & $70^{\circ}$ & 116.7 \\
\hline
\end{tabular}

Auf diesen Daten, kann die Beimengung von Cerylalkohol und Myricylalkohol in diesen Wachsalkohol vermutet werden.

5.) Kohlenwasserstoff des unverseifbaren Bestandteils.

Die Kohlenwasserstoffe wurden gereinigt zuerst durch mehrfaches Kochen mit dem Gemische von gleichen Volumen von Amylalkohol und rauchenden Salzsäure. Es ist unlöslich in siedendem Essigsäureanhydrid und Alkohol, löslich in warmen Aether, Benzol und Chloroform. Es schmilzt bei $68^{\circ}$ (unkorr.). Die Elementarzusammensetzung ist, $\mathrm{C} \%$ 84.11, $\mathrm{H} \% 14.84$.

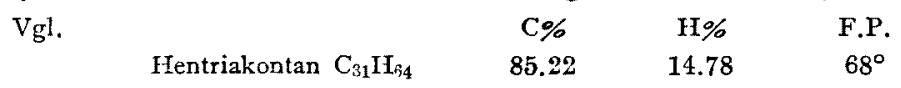

Es addiert sich nicht Brom. Wir vermuteten deswegen, dass der Wachskoholenwasserstoff hauptsächlich aus Hentriakontan bestand.

6.) Die Fettsäuren vom Kokonwachs.

a) Die Menge der gesamten Fettsäuren kann aus der Differenz von reinem Wachs und dessen unverseifbaren Bestandteil erhalten werden : es betrug also $36.86 \%$ des Wachses. Wir berechneten aus der Verseifungszahl und den gesamten Fettsäuren,

Neutralisationszahl 300.52, Molekulargewicht 186.71.

b) Die Hehnerschen Zahlen betrugen $13.40 \%$. Die in Wasser unlöslichen Fettsäuren zeigten folgende Eigenschaften:

Schmelzpunkt $85^{\circ}$ (unkorr.), Neutralisationszahl 126.74, Molekulargewicht 442.72, Elmentarzusammensetzung $\mathrm{C} \%$ 79.96, $\mathrm{H} \% 14.04$.

$\begin{array}{lcccc}\text { Vgl. } & \mathrm{C} \% & \mathrm{H} \% & \text { F. P. } & \text { Mol.-gew. } \\ \text { Melissinsäure } & 79.56 & 1337 & 88.5^{\circ} & 452.48\end{array}$

Deshalb kann das Vorhandensein von Melissinsäure vermutet werden, jedoch muss die Anwesenheit von populären Fettsäuren z. B. Palmitin- und 
Stearinsäure als zweifelhaft angesehen werden.

c) Die in Wasser löslichen Fettsäuren betrugen $63.65 \%$ der gesamten Fettsäuren. Die saure Lösung, welche von in Wasser unlöslichen Fettsäuren getrennt wurde, hat einen stark riechenden, unangenehmen Geruch und bewies deutlich das Vorhandensein von niedrigen Fettsäuren.

Wir berechneten, Neutralisationszahl 399.76, Molekulargewicht 140.36 .

Aus den vorhergehenden Tatsachen fanden wir, dass diese Kokonwachse viel Ähnlichkeit mit den Bienen-wachsen haben.

\title{
ON THE SIMPLIFIED, SATURATED CALOMEL ELECTRODE. REPORT II.
}

By

\author{
Arao Itano, Ph. D. \\ (Received Feb. 2nd., 1929).
}

This paper deals with a simplified, portable form of saturated calomel electrode which is arranged in an ordinary vessel and has a certain advantages over the Ostwald vessel, and serves the purpose satisfactorily.

The mixture of mercury, calomel and saturated potassium chloride is placed in an ordinary pyrex glass $c$ up in which a platinum electrode and one end of an agar bridge are placed properly through a rubber stopper, and the other end of agar bridge is immersed in the saturated $\mathrm{KCl}$ solution by which the chain is formed.

The calomel electrode thus prepared gives very good results in comparison with those obtained using the standard $\mathrm{N} / 10 \mathrm{KCl}$ calomel electrode. Some of the results are shown in Table I.

\section{Tabele I.}

$\mathrm{pH}$ determined by the simplified and standard Calomel Flectrode.

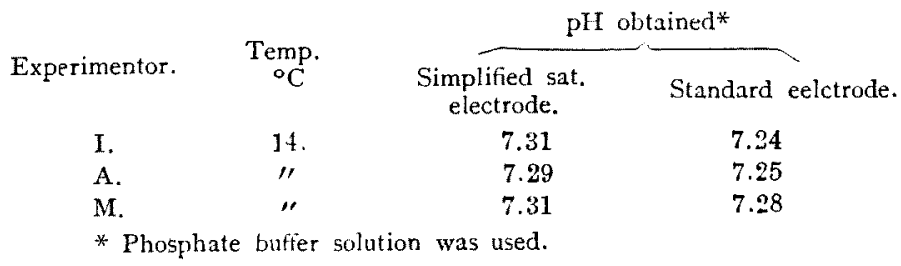

